\title{
Sublingual vs Oral Misoprostol for Labor Induction
}

\author{
${ }^{1}$ Sujata Siwatch, ${ }^{2}$ Goter Doke, ${ }^{3}$ Jasvinder Kalra, ${ }^{4}$ Rashmi Bagga
}

\section{ABSTRACT}

Background: This study compares the efficacy and safety of sublingual vs oral misoprostol for induction of labor.

Materials and methods: 160 women admitted for induction of labor at the Postgraduate Institute of Medical Education and Research, Chandigarh were randomized to receive $25 \mu \mathrm{g}$ misoprostol orally 3 hourly or $25 \mu \mathrm{g}$ sublingual misoprostol 4 hourly for labor induction.

Outcome: The two groups were compared for number of women not delivered in 24 hours, misoprostol dose required, induction delivery interval, incidence of uterine contraction abnormalities, mode of delivery, side effects and neonatal outcomes.

Results: Low dose of misoprostol is efficacious with both routes of administration. Majority women delivered vaginally and of them, comparable numbers in both vaginal and sublingual misoprostol groups delivered within 24 hours of induction (93.1 and $83.7 \%)$. The sublingual route is associated with a statistically significant lesser induction to delivery interval (14.8 \pm 6.2 hours vs $17.67 \pm 7.32$ hours) and lesser requirement of oxytocin augmentation (62.5 vs $35 \%$ ). The occurrence of uterine contraction abnormalities and neonatal outcome was similar in both groups.

Conclusion: The low dose of $25 \mu \mathrm{g}$ is efficacious and safe by both sublingual and oral routes. Sublingual route has lesser induction to delivery interval and lesser requirement for oxytocin augmentation.

Keywords: Misoprostol, Labor induction, Sublingual, Oral.

How to cite this article: Siwatch S, Doke G, Kalra J Bagga R. Sublingual vs Oral Misoprostol for Labor Induction. J Postgrad Med Edu Res 2014;48(1):33-36.

Source of support: Nil

Conflict of interest: None

\section{INTRODUCTION}

Misoprostol, a prostaglandin E1 analog is a popularly used cervical ripening and inducing agent. It can be administered by various routes that include oral, buccal, sublingual and

${ }^{1}$ Pool Officer, ${ }^{2}$ Junior Specialist, ${ }^{3,4}$ Professor

1,3,4 Department of Obstetrics and Gynecology, Postgraduate Institute of Medical Education and Research, Chandigarh, India

${ }^{2}$ Department of Obstetrics and Gynecology, Arunachal Pradesh Health Services, General Hospital, Aalo, Arunachal Pradesh, India

Corresponding Author: Sujata Siwatch, Pool Officer Department of Obstetrics and Gynecology, Postgraduate Institute of Medical Education and Research, Chandigarh India, Phone: 01722756331, e-mail: siwatch1@yahoo.com vaginal routes. ${ }^{1-5}$ The efficacy may differ with the routes of administration due to difference in the pharmacokinetics. The sublingual and vaginal routes bypass the enterohepatic circulation and the vaginal route is thought to have a local effect as well. Oral and sublingual routes are however more acceptable due to ease of administration. There have been few studies comparing the efficacy of low dose sublingual and oral misoprostol for labor induction. ${ }^{2-5}$

The present study was planned to evaluate the efficacy and suitability of sublingual misoprostol for induction of labor when compared with an equivalent low dose (25 mg) given vaginally. Clearance from the Ethics Committee of the institute was taken. Considering the vaginal delivery rate of $73.8 \%$ with sublingual misoprostol by Shetty et $\mathrm{al}^{1}$, a study with $80 \%$ power and $\alpha$ error of $5 \%$ required a sample size of 138 women to detect a difference of $20 \%$ between the two groups.

\section{PATIENTS AND METHODS}

A total of 160 pregnant women admitted in the labor room in Postgraduate Institute of Medical Education and Research, Chandigarh, India, for induction of labor over a 2 years period were recruited in the study, provided they fulfilled the following inclusion and exclusion criteria and were willing to participate in the study.

Inclusion criteria included pregnancy with singleton live fetus with cephalic presentation, gestational age $\geq 32$ weeks, Bishop's score $<5$. Exclusion criteria included previous uterine surgery, major fetal malformation, parity $>3$, antepartum hemorrhage, severe renal, hepatic failure, any medical disorder as cardiac disease, glaucoma, convulsive disorder, asthma, severe anemia, any contraindication to vaginal delivery like cephalopelvic disproportion, severe IUGR, oligohydramnios ( $<5$ centimeters), clinically suspected chorioamnionitis or history of unclean vaginal examination, known hypersensitivity to prostaglandin, any oral pathology and acid peptic disease.

On admission, a detailed history of present pregnancy, past medical and obstetric history was taken. A general physical examination and an obstetrical examination were done to ascertain fundal height, lie and presentation of the fetus and the Bishop's score. An informed consent was taken from the subjects willing to participate in the study. They were then allocated according to a computer generated randomization chart into 2 groups of 80 women each for 
labor induction. Group 1 received $25 \mu \mathrm{g}$ of misoprostol sublingually 4 hourly up to a maximum of six doses. Group 2 received $25 \mu \mathrm{g}$ of misoprostol orally 3 hourly up to a maximum of eight doses. The recruitment was done by the investigator or resident in the labor room. Misoprostol used was $1 / 4$ th tablet of 100 microgram misoprostol (Cytotec).

A vaginal examination was performed to assess the Bishop's score followed by administration of $25 \mu \mathrm{g}$ of misoprostol, vaginally in group 1 and sublingually in group 2. Progress of labor and fetal heart rate pattern was monitored and subsequent doses of misoprostol given every 4 hours until there were regular uterine contractions (i.e. 3 contractions every 10 minutes), cervical dilatation $>3 \mathrm{~cm}$, Bishop score of $>8$ or evidence of tachysystole, hypertonus or hyperstimulation. In the event of tachysystole, uterine hypertonus or hyperstimulation, the woman was placed in left lateral position and oxygen was administered by facemask at the rate of 8 liters per minute. Injection terbutaline $250 \mu \mathrm{g}$ was given by subcutaneous route in case of hyperstimulation and hypertonus. The uterine contraction was monitored by palpation and fetal heart rate was monitored by intermittent auscultation as per protocol. Cardiotocographic tracing was taken in the event of features suggestive of fetal distress. With nonreassuring fetal heart rate tracing, further doses were withheld. Once in active phase of labor, routine intrapartum management was performed as per the protocol of the labor room. If the patient was undelivered at the end of 24 hours, the decision about further management was as per the clinician in charge. The presence of any side effect (fever, gastrointestinal side effects) was noted.

Primary outcome of the study was percentage of women delivering vaginally. Secondary outcomes included total number of doses of $25 \mu \mathrm{g}$ of misoprostol required for labor induction, incidence of vaginal delivery within 24 hours, induction delivery interval, change in Bishop's score after misoprostol, number of patients requiring oxytocin augmentation, total oxytocin dose required, mode of delivery, rate of uterine tachysystole, uterine hypertonus and hyperstimulation, fever and gastrointestinal side effects, neonatal Apgar score and admission to neonatal intensive care unit.

\section{STATISTICAL ANALYSIS}

Statistical analysis was done by using Chi-square test, student $\mathrm{t}$ - test and Fisher Exact test by SPSS software (version 15.0). Intention to treat analysis was done. However, there were no dropouts.

\section{RESULTS}

One hundred and sixty women were recruited after consent and randomized into sublingual and vaginal misoprostol groups. Intention to treat analysis was used. There were no dropouts in the study. The demographic features, indications for induction and preinduction scores were comparable between the two arms (Table 1). The mean age of the women was $25.19 \pm 3.41$ years in group 1 and $25.2 \pm 3.48$ years in group 2 . The majority of participants were primiparous $(75.6 \%)$ and at term gestation $(77.5 \%)$. The mean preinduction Bishop's score at the start of induction was comparable in both groups, being $2.31 \pm 0.793$ in group 1 and $2.19 \pm 0.920$ in group 2 .

Table 2 shows the outcome of labor and fetal outcomes in the two groups. Majority of women in both groups delivered vaginally $(91.25 \%$ in sublingual misoprostol and $92.5 \%$ in oral misoprostol group). Among those who delivered vaginally, comparable number of women in both vaginal and sublingual misoprostol groups delivered within 24 hours of induction (93.1 and 83.7\%). Mean induction delivery was significantly less in group 1 compared to group $2(14.8 \pm 6.2$ hours $v s 17.67 \pm 7.32 \mathrm{hrs})$. Significantly lesser number of total, nulliparous and multiparous women in group 1 required oxytocin for augmentation as compared to group $2(62.5,41,65$ vs $35 \%, 15.8 \%$ and $55 \%)$. Notably, the mean dose of oxytocin required in both groups was comparable. The majority of women required up to three doses of misoprostol (mean dose 2.6 and 3.4 respectively). However, significantly more women $14 / 80$ (17.5\%) in sublingual group required only a single dose of misoprostol as compared to the oral misoprostol group 2/80 (2.5\%) $(p=0.0002)$. The incidence of abnormal uterine contraction (tachysystole and hyperstimulation) was low and comparable in both the groups. None of the women had prolonged third

Table 1: The demographic profile of subjects in the study was comparable between the sublingual (group 1) and oral (group 2) misoprostol groups

\begin{tabular}{lllcc}
\hline & Group 1 $(n=80)$ & Group 2 $(n=80)$ & Total $(n=160)$ & $p$-value \\
\hline Age in years (mean \pm SD) & $25.19 \pm 3.41$ & $25.2 \pm 3.48$ & - & 0.985 \\
Nullipara & $61(76.0 \%)$ & $60(75.0 \%)$ & $121(75.6 \%)$ & 0.862 \\
Period of gestation in weeks (mean \pm SD) & $37.26 \pm 2.2$ & $37.39 \pm 206$ & - & 0.700 \\
Induction for premature rupture of membranes & $27(33.8 \%)$ & $35(43.8 \%)$ & $62(38.8 \%)$ & 0.194 \\
Induction for hypertensive disorders & $27(33.8 \%)$ & $15(33.8 \%)$ & $39(24.4 \%)$ & $0.031^{*}$ \\
Mean preinduction score & $2.31+0.793$ & $2.19+0.920$ & - & 0.378 \\
\hline
\end{tabular}

*Significant difference, $p<0.05$ but $>0.01$ 
Table 2: Characteristics and outcome of labor between the sublingual (group 1) and oral (group 2) misoprostol groups

\begin{tabular}{|c|c|c|c|}
\hline & Group $1(n=80)$ & Group $2(n=80)$ & $p$-value \\
\hline Vaginal delivery & $73(\%)$ & $74(\%)$ & 0.777 \\
\hline No vaginal delivery within 24 hours & $5(6.8 \%)$ & $12(16.2 \%)$ & 0.072 \\
\hline Duration of labor & $\begin{array}{l}0-12 \text { hrs } 31(42.5 \%) \\
0-24 \text { hrs } 68(93.1 \%)\end{array}$ & $\begin{array}{l}0-12 \text { hrs } 19(25.7 \%) \\
0-24 \text { hrs } 62(83.7 \%)\end{array}$ & $\begin{array}{l}0.041^{*} \\
0.224\end{array}$ \\
\hline \multirow[t]{3}{*}{ Mean induction delivery interval } & $14.8 \pm 6.2$ & $17.67 \pm 7.32$ & 0.008 \\
\hline & $\mathrm{N}-16.04 \pm 6.0$ & $\mathrm{~N}-19.02 \pm 7.46$ & 0.006 \\
\hline & $M-11.29 \pm 5.49$ & $M-14.03 \pm 5.58$ & $0.002^{* *}$ \\
\hline \multirow[t]{2}{*}{ DROM } & With PROM $22.43 \pm 16.85$ & With PROM $23.23 \pm 1.29$ & 0.673 \\
\hline & Without PROM $6.14 \pm 8.65$ & Without PROM $8.91 \pm 5.34$ & $0.016^{*}$ \\
\hline Cesarean section & $7(8.8 \%)$ & $6(7.5 \%)$ & 0.777 \\
\hline \multicolumn{4}{|l|}{ Indication for cesarean section } \\
\hline MSL & 3 of 7 & 2 of 6 & 1 \\
\hline Cord presentation & 1 & - & 1 \\
\hline Fetal bradycardia & 3 & 3 & 1 \\
\hline NPL & - & 1 & 0.999 \\
\hline Mean doses of $25 \mu \mathrm{g}$ misoprostol used & 2.6 & 3.4 & 1 \\
\hline Mean Bishop's score after 2 doses of misoprostol & $6.41 \pm 1.28$ & $5.61 \pm 1.55$ & $0.0005^{* *}$ \\
\hline $\begin{array}{l}\text { Mean change in Bishop's score after } 2 \text { doses of } \\
\text { misoprostol }\end{array}$ & $4.09 \pm 1.32$ & $3.42 \pm 1.36$ & $0.0019^{* *}$ \\
\hline \multirow[t]{3}{*}{ Women requiring oxytocin } & $28(35 \%)$ & $50(62.5 \%)$ & 0.0005 \\
\hline & $\mathrm{N}-25 / 61(41 \%)$ & $N-39 / 60(65 \%)$ & $0.0082^{* *}$ \\
\hline & $M-3 / 19(15.8)$ & $\mathrm{M}-11 / 20(55 \%)$ & $0.0265^{*}$ \\
\hline \multirow[t]{3}{*}{ Mean dose of oxytocin required } & $38.0 \pm 22.03$ & $40.24 \pm 22.5$ & 0.525 \\
\hline & $\mathrm{N}-38.4 \pm 21.87$ & $\mathrm{~N}-44.6 \pm 22.1$ & 0.076 \\
\hline & $M-34.6 \pm 28.09$ & $M-25.0 \pm 17.6$ & $0.0105^{*}$ \\
\hline \multicolumn{4}{|l|}{ Complications } \\
\hline Hypertonus & - & - & - \\
\hline Tachysystole & $1(1.3 \%)$ & $1(1.3 \%)$ & 1.0 \\
\hline Hyperstimulation & $1(1.3 \%)$ & $1(1.3 \%)$ & 1.0 \\
\hline Terbutaline use & $1(1.3 \%)$ & $1(1.3 \%)$ & 1.0 \\
\hline Meconium stained liquor & $3(3.8 \%)$ & $2(2.5 \%)$ & 1 \\
\hline Third stage complications cervical tear & $1(1.3 \%)$ & - & 1 \\
\hline Postpartum hemorrhage & - & - & - \\
\hline Vomiting, nausea & $4(5 \%)$ & $5(6.3 \%)$ & 1 \\
\hline \multicolumn{4}{|l|}{ Neonatal outcomes } \\
\hline Mean birth weight $(\mathrm{kg})$ & $2.55 \pm 0.51$ & $2.62 \pm 0.54$ & 0.401 \\
\hline \multicolumn{4}{|l|}{ Mean Apgar score (AS) } \\
\hline 1 minute & $7.99 \pm 0.56$ & $7.93 \pm 0.44$ & 0.452 \\
\hline 5 minutes & $8.99 \pm 0.52$ & $9.00 \pm 0.42$ & 0.894 \\
\hline Neonatal death & - & - & - \\
\hline Neonatal hyperbilirubinemia & $8(10 \%)$ & $8(10 \%)$ & 1 \\
\hline
\end{tabular}

DROM: Duration of rupture of membranes; PROM: Premature rupture of membranes; N: Nulligravida; M: Multigravida; MSL: Meconium stained liquor; NPL: Nonprogress of labor; HR: Hours; *Significant difference, $p<0.05$ but $>0.01$; ${ }^{* *}$ Very significant difference, $p<0.01$

stage, postpartum hemorrhage or retained placenta. Only 1 woman from group 1 had a cervical tear. Neonatal outcome in terms of birth weight, Apgar score at 1 and 5 minutes, cord $\mathrm{pH}$ and admission and stay in neonatal intensive care unit was comparable in both the groups.

\section{DISCUSSION}

Misoprostol is a promising prostaglandin which had been widely used for inducing abortions, management of postpartum hemorrhage and even for induction of labor. ${ }^{1}$ Though it is not FDA approved for labor induction and is contraindicated for use in pregnancies with previous scarred uterus owing to risk of uterine rupture, its off-label use for labor induction has gained popularity as it is cheap, easily stored, and effective with few side effects. It acts to promote both cervical ripening and uterine contractions and can be administered by various routes like oral, vaginal, sublingual, buccal and rectal. ${ }^{2,3}$

Pharmacokinetic studies on various routes of administration of misoprostol have shown that sublingual and oral routes have a quicker onset of action. ${ }^{6,7}$ The peak levels were highest in sublingual group as compared to oral group, 
probably due to the avoidance of first hepatic pass metabolism in the sublingual group. ${ }^{6}$ However, sublingual and oral routes are preferred by patients due to ease of administration. Sublingual and oral routes are also preferred in cases of rupture of membranes where vaginal route may not be an effective method of administration due to local wash away effect of liquor on the misoprostol.

There are few studies that compare the safety and effectiveness of sublingual and oral misoprostol for labor induction. Muzonzini G, in the Cochrane review in 2010, reviewed 2 trials by Shetty et al with equal doses (fifty micrograms) administered by both routes..$^{1-3}$ They concluded that the sublingual route was associated with more women delivering within 24 hours of induction with less oxytocin augmentation but these results were not statistically significant. ${ }^{3}$ There was no difference in the outcome when higher dose was used orally, i.e. fifty microgram sublingually and hundred microgram orally. ${ }^{1}$ In another study by Malik et al that compared induction of labor in women with term premature rupture of membranes using $100 \mu \mathrm{g}$ of oral misoprostol or $50 \mu \mathrm{g}$ sublingual misoprostol administered every 4 hourly, more women, i.e. $92 \%$ women delivered vaginally in 12 hours in sublingual group as compared to $84 \%$ in the oral group $(\mathrm{p}<0.05) .{ }^{4}$ In our study also, the difference in women delivering in 24 hours of induction was not statistically different. ${ }^{4}$ Our study participants included women with preterm gestations after 32 weeks as compared to term gestation patients in previous studies. Though in our study, a lower dose of $25 \mu \mathrm{g}$ misoprostol was used in both groups; the mean induction delivery interval was shorter than in the study by Shetty et al. ${ }^{1}$ The mean induction delivery interval was statistically lesser in the sublingual as compared to the oral group. Sixty four and thirty two percentage of women in sublingual and oral groups respectively delivered after single dose in the study by Malik et al as compared to 17.5 and $2.5 \%$ in our study which is due to the lower dose used in our study. ${ }^{4}$ The mean preinduction Bishop's score was somewhat higher in the study by Shetty et al $(3.7 \pm 1.5$ in sublingual group and $3.6 \pm 1.6$ in oral group) as compared to our study. ${ }^{2}$ Our study also analyzed the effect of misoprostol on cervical ripening using change in Bishop's score after 2 doses of misoprostol. There were few and comparable number of rates of hyperstimulation or tachysystole in our study as in previous studies. ${ }^{3-5}$ Shetty et al in their study noted vomiting in $12 \%$ each in sublingual and oral groups, which is more than our study. ${ }^{1}$ This could be because of higher misoprostol dose $(50 \mu \mathrm{g})$ in their study. The neonatal outcomes were also comparable in the two groups.

The study has its limitations due to the nonfeasibility of blinding of subjects and researcher to the mode of administration of the drug. Body mass index may determine the dose of misoprostol required for induction. Future research may suggest body mass index to titrate the dose requirements of misoprostol for labor induction.

\section{CONCLUSION}

The study concludes that both oral as well as sublingual misoprostol are safe and effective methods of labor induction in women with unfavorable cervix in third trimester of pregnancy and that a low dose of $25 \mu \mathrm{g}$ sublingual or oral misoprostol is also as effective as reported with a higher dose in the literature. The sublingual route is associated with a statistically significant lesser induction to delivery interval and lesser requirement of oxytocin augmentation. Lesser number of doses of sublingual misoprostol was needed for establishing regular contractions as compared to oral group. However, a larger number of women need to be studied in order to achieve a statistical power sufficient to compare the occurrence of infrequent events.

\section{REFERENCES}

1. Shetty A, Danielian P, Templeton A. Sublingual misoprostol for the induction of labor at term. Am J Obstet Gynecol 2002;186: 72-76.

2. Shetty A, Mackie L, Danielian P, Rice P, Templeton A. Sublingual compared with oral misoprostol in term labour induction: randomized controlled trial. Br J Obstet Gynecol 2002;109: 645-650.

3. Muzonzini G, Hofmeyr GJ. Buccal or sublingual misoprostol for cervical ripening and induction of labour. Cochrane Database Syst Rev 2004 Oct 8;(4):CD004221.

4. Malik HZ, Khawaja NP, Zahid B, Rehman R. Sublingual versus oral misoprostol for induction of labour in prelabour rupture of membranes at term. J Coll Physicians Surg Pak 2010 Apr;20(4):242-245.

5. Elhassan EM, Nasr AM, Adam I. Sublingual compared with oral and vaginal misoprostol for labor induction. Int J Gynaecol Obstet 2007 May;97(2):153-154.

6. Zieman M, Fong SK, Benowitz NL, Banster D, Darney PD. Absorption kinetics of misoprostol with oral and vaginal administration. Obstet Gynecol 1997;90:88-92.

7. Abdel-Aleem H, Villar J, Gulmezoglu AM, Mostafa SA, Youssef AA, Shokry M, Watzer B. The pharmacokinetics of the prostaglandin E1 analogue misoprostol in plasma and colostrums after postpartum oral administration. Eur J Obstet Gycecol Reprod Biol 2003;108:25-28. 